\title{
Detecting unstable structures and controlling error growth by assimilation of standard and adaptive observations in a primitive equation ocean model
}

\author{
F. Uboldi ${ }^{1}$ and A. Trevisan ${ }^{2}$ \\ ${ }^{1}$ no current affiliation \\ ${ }^{2}$ CNR-ISAC, Bologna, Italy \\ Received: 28 June 2005 - Revised: 11 January 2006 - Accepted: 11 January 2006 - Published: 24 March 2006
}

\begin{abstract}
Oceanic and atmospheric prediction is based on cyclic analysis-forecast systems that assimilate new observations as they become available. In such observationally forced systems, errors amplify depending on their components along the unstable directions; these can be estimated by Breeding on the Data Assimilation System (BDAS). Assimilation in the Unstable Subspace (AUS) uses the available observations to estimate the amplitude of the unstable structures (computed by BDAS), present in the forecast error field, in order to eliminate them and to control the error growth. For this purpose, it is crucial that the observational network can detect the unstable structures that are active in the system. These concepts are demonstrated here by twin experiments with a large state dimension, primitive equation ocean model and an observational network having a fixed and an adaptive component. The latter consists of observations taken each time at different locations, chosen to target the estimated instabilities, whose positions and features depend on the dynamical characteristics of the flow. The adaptive placement and the dynamically consistent assimilation of observations (both relying upon the estimate of the unstable directions of the data-forced system), allow to obtain a remarkable reduction of errors with respect to a non-adaptive setting. The space distribution of the positions chosen for the observations allows to characterize the evolution of instabilities, from deep layers in western boundary current regions, to near-surface layers in the eastward jet area.
\end{abstract}

\section{Introduction}

The growth of errors in nonlinear dynamical systems such as the ocean and the atmosphere is governed by the trajectory instability that characterizes chaos. Errors of various origin, if they are not too large, eventually grow or decrease following the unstable directions, that characterize the local geom-

Correspondence to: F. Uboldi

(uboldi@magritte.it) etry of the attractor. As a consequence, in cyclic observationanalysis-forecast systems, the forecast error has an important component on the unstable subspace, while other "unstructured" components are due to errors that have been introduced recently. When correcting a model state by assimilating observations, if a suitable estimate of the unstable directions is available, this information can, and should, be used to constrain the analysis increment. A direct way to do this is to introduce the information on the unstable directions in the estimate of the forecast error covariance matrix, that constitutes the prior information in the analysis.

The present work focuses on the unstable components of the forecast error, estimated by a modified form of breeding (Sect. 2.1) that accounts for "observational forcing" introduced at the analysis step. It is shown that the growth of errors can be controlled by using this information: 1) to choose the locations of adaptively taken observations and 2) in the assimilation (Sect. 2.2) of all the available (standard and adaptive) data. These principles are demonstrated here in the context of a primitive equation ocean system with a large state dimension.

Data assimilation in the ocean accounts for the dynamics in variational schemes (Talagrand and Courtier, 1987; Bennett, 1992, 2002; Wunsch, 1996) and in sequential schemes that, starting from statistical principles, allow for dynamical evolution of error statistics (Ghil, 1989, 1997; Evensen, 1994, 2003, 2004; Burgers et al., 1998; Brasseur et al., 1999; Pham, 2001). Recent joint efforts have been devoted to obtain four-dimensional analysis of the ocean flow, by applying sophisticated assimilation techniques (ECCO Consortium, 1999). On the other hand, many operational forecasting centers still use assimilation schemes that are based on stationary prior statistics, for simplicity of implementation and computer time constraints, even if much work is put into refining the agreement with statistics based on models and real data (De Mey and Benkiran, 2002), and work is generally under way to implement dynamically evolving covariances.

Recently, Zang and Malanotte-Rizzoli (2003) have shown how dynamically evolving covariances, as estimated by an 
Ensemble Kalman Filter (EnKF), are able to account for chaotic regime transitions, thus outperforming stationary schemes such as Reduced Rank Extended Kalman Filter and Optimal Interpolation (OI). In their work, the two latter schemes behave similarly, only marginally improving the saturated freely evolving errors in a low-predictability system configuration, while only in a configuration that is highly predictable the stationary schemes are able to reduce the errors in a satisfactory way.

The concept of adaptive observations is particularly interesting for the ocean, where an important part of the observations (vertical profiles and Lagrangian buoys) have always been taken by mobile systems (oceanographic ships), in contrast with the fixed network of radiosondes existing for the atmosphere, which is mainly based on land. It is then appropriate to study how the locations of oceanographic observations can be chosen depending on the dynamical situation.

For the atmosphere, much of the work on "adaptive" or "targeted" observations has been based on the concept that targeting aims at reducing the forecast error inside a "verification" area, at a certain "verification" time. Area and time are selected on the basis, on the one hand, of the meteorological phenomena, and, on the other hand, of their predicted impact on human activities - in particular those of interest for financing institutions and countries.

Different targeting strategies have been proposed and tested by means of experimental campaigns. Methods based on Singular Vector Decomposition (SVD) (Palmer et al., 1998; Bergot et al., 1999) of the tangent linear model estimate "sensitive" regions, where inaccuracies present in the analysis are foreseen to amplify the most, resulting in a degradation of the forecast at the verification area and time. SVD calculations are performed with different norms (in essence, scalar products), mainly the total energy norm and the "Hessian" (of the variational cost function) norm, which is an approximation, based on the (stationary) prior covariances, to the inverse analysis error covariance.

The complete and time-evolving inverse analysis error covariance has been proposed as the ideal initial time norm for SVD by Ehrendorfer and Tribbia (1997). SVD calculations with a norm based on a dynamically evolving covariance from an EnKF have been performed by Buehner and Zadra (2006). Recent results from the 2003 North Atlantic THORPEX Regional field Campaign (NA-TReC) show that, still, there are significant cases of degradation of the forecast, presumably depending, apart from "operational" limitations like imprecise deployments and observational errors, on how the supplementary data have been assimilated (stationary prior statistics?). Fourrié et al. (2006) analyzed in detail the results of the NA-TReC with the French operational model ARPEGE and discussed the impact of the different types of supplementary observations.

In the Ensemble Transform Kalman Filter (ETKF) approach (Bishop et al., 2001) the adaptive observations are deployed with the aim of minimizing a measure of the forecast error covariance matrix, estimated at a verification forecast time (successive to observation time) and geographically localized inside a verification region. The estimate of the covariance matrix is ensemble-based and the minimization is realized with respect to a set of possible observation deployments (i.e. a set of observation operators), by computing how the ensemble members are "transformed" through the analysis step, before the model integration to verification time. ETKF has also been employed in the NA-TreC and in the Winter Storm Reconnaissance Program (WSRP) (Szunyogh et al., 2000, 2002): preliminary results showed some improvements, but degradation cases still exist.

With regard to the ocean, there exist recent studies on the impact of different possible future satellite "constellations" on oceanic assimilation systems (Mourre, 2004; Mourre et al., $2006^{1}$ ). Köhl and Stammer (2004) studied optimal locations, using an inverse (adjoint) method in which the observational increments are the control variables.

In the present work, targeting is not focused on improving the forecast at a particular location and time. Rather, the assimilation of supplementary observations is aimed at keeping the trajectory of the cyclic observation-assimilation-forecast system close to the real system trajectory. A major improvement in the control of errors derives from the combination of targeting strategies and dynamically consistent assimilation; the benefit of adaptively located observations is greatly enhanced if their positions and their assimilation are designed in order to target the instabilities; the estimate of unstable directions is based on BDAS (Breeding on the Data Assimilation System), and AUS (Assimilation in the Unstable Subspace) is such that the analysis update has locally the same structure as that of the unstable vector whose maximum is in the location chosen for the adaptive observation.

The basis of the BDAS-AUS method used here have been introduced by Trevisan and Uboldi (2004, hereafter TU04), who applied it to a small chaotic (and noisy) system (Lorenz and Emanuel, 1998). Since then, applications to more complex models have been considered (Uboldi et al., 2005, hereafter UTC05). Complete results obtained with the quasigeostrophic atmospheric model of Rotunno and Bao (1996), also in presence of observational errors, are presented in a recent work (Carrassi et al., 2006 ${ }^{2}$, hereafter CTU06). Experience showed that each application requires specific implementation choices, but that the method is sufficiently flexible and robust to be successfully applied to different contexts: models of various complexity and in the presence of observational and model error. In the present work the focus is on a complex, primitive equation ocean model with a large number of unstable directions. Experiments are done in a perfect model, perfect observations setting: this is of course a limitation, because realistic models and real observations

\footnotetext{
${ }^{1}$ Mourre, B., De Mey, P., Ménard, Y., Lyard, F., and Le Provost, C.: Relative performances of future altimeter systems and tide gauges in controlling a model of the North Sea high frequency barotropic dynamics, Ocean Dynamics, in review, 2006.

${ }^{2}$ Carrassi, A., Trevisan, A., and Uboldi, F.: Adaptive observations and assimilation in the unstable subspace by breeding on the data-assimilation system, Tellus, submitted, 2006.
} 
are affected by errors; on the other hand, that of perfect settings is a necessary and meaningful step, because it allows to clarify some theoretical aspects, and to highlight the mechanism of error growth, rather than the various sources of error.

Details of the technique used to estimate the (local structures associated with the) unstable directions depend on the characteristics of the system. First of all, the number of unstable vectors and their growth rates characterize each freely evolving system. Moreover, independent unstable structures may appear well separated in the geographical space, or, in contrast, they may be concentrated and overlapping in the same region (where a front is located, for example). If a fixed observational network is present, then its features may have an influence on the estimate of instabilities: a network of fixed vertical profiles based on land for atmospheric observations provide an "observational forcing" (TU04, see also Sect. 2 below) that is different from that provided on an ocean system by satellite sea surface height ( $\mathrm{SSH}$ ) observations, available under satellite tracks that cover a wide horizontal domain, but without direct information on the vertical structure of the ocean. The use of adaptive observations and their type (profiles, floating buoys, adaptable remote systems) contribute to change the characteristics of the instabilities that appear (survive) in the forced system. Finally, these are also influenced by the characteristics of the assimilation scheme, through which the observational forcing enters the system.

In this work it is shown that it is possible to estimate the (local) unstable structures that are present in a primitive equation ocean system, so that, if the observational network, in its fixed and adaptive components, is able to detect them, then the assimilation of observations is successful in controlling the error growth.

The assimilation method, already presented in the authors' previous works, is described in Sect. 2. The ocean system and a standard assimilation scheme are presented in Sect. 3 . Section 4 describes the choices made in the implementation of BDAS, and summarizes some results obtained using the fixed observational network, already included in UTC05. Section 5 deals with implementation choices and analysis of results obtained by combining the standard network with adaptive observations, assimilated by AUS. Conclusions are summarized in Sect. 6.

\section{Assimilation in the unstable subspace of the data forced system.}

2.1 Estimating the unstable directions by Breeding on the Data Assimilation System (BDAS)

A forecast state is obtained by integrating the model from a previous analysis state:

$\mathbf{x}_{k+1}^{f}=\mathcal{M}\left(\mathbf{x}_{k}^{a}\right)$
The (tangent linear) perturbative equation relative to Eq. (1) is:

$\delta \mathbf{x}_{k+1}^{f}=\mathbf{M} \delta \mathbf{x}_{k}^{a}$

where $\mathbf{M}$ is the Jacobian of $\mathcal{M}$. A small perturbation of the state evolves and, if kept small, progressively acquires the structure of a linear combination of the unstable directions. The breeding method computes these directions by integrating the nonlinear model on a control state and on one (or more) perturbed states, and periodically renormalizing the amplitude of the perturbations to a "small" value. In atmospheric applications, the normalization amplitude and frequency and the length of the complete breeding period may be adjusted in order to filter out fast-growing convective instabilities that quickly saturate to small energy values, and to select, in this way, baroclinic scale instabilities (Toth and Kalnay, 1997; Kalnay, 2003).

When observations are available, an analysis step is performed:

$\mathbf{x}_{k+1}^{a}=\mathbf{x}_{k+1}^{f}-\mathbf{K} H\left(\mathbf{x}_{k+1}^{f}\right)+\mathbf{K y}_{k+1}^{o}$

where $H$ is the (possibly nonlinear) observation operator and $\mathbf{K}$ is the gain matrix, linearly relating the analysis increment $\mathbf{x}^{a}-\mathbf{x}^{f}$ to the innovation $\mathbf{y}^{o}-H\left(\mathbf{x}^{f}\right)$. This expression, typical of schemes such as Optimal Interpolation and Kalman Filters (analysis step), may also account for 3D-Var schemes in which the observation operator $H$ is linearized on the forecast state. The same expression may also represent a linearized (or incremental) 4D-Var scheme assimilating observations distributed in a time interval, by extending the concept of observation operator to include a model integration: on this point, see the book by Bennett (2002), or, for a discrete formulation, Uboldi and Kamachi (2000).

By substituting Eq. (1) in Eq. (3), the evolution equation between two successive analysis states (the same can be done for two successive forecast states) is obtained:

$\mathbf{x}_{k+1}^{a}=\mathcal{M}\left(\mathbf{x}_{k}^{a}\right)-\mathbf{K} H\left[\mathcal{M}\left(\mathbf{x}_{k}^{a}\right)\right]+\mathbf{K y}_{k+1}^{o}$

This is the equation of the system forced by the cyclic assimilation of observations.

If $\eta^{a}$ is the analysis error, its first-order evolution from analysis step $k$ to analysis step $k+1$ is:

$\boldsymbol{\eta}_{k+1}^{a}=(\mathbf{I}-\mathbf{K H}) \mathbf{M} \boldsymbol{\eta}_{k}^{a}+(\mathbf{I}-\mathbf{K H}) \boldsymbol{\eta}_{k+1}^{\mathcal{M}}+\mathbf{K} \boldsymbol{\varepsilon}_{k+1}^{o}$

where $\mathbf{H}$ is the Jacobian of $H$ and $\mathbf{I}$ is the identity matrix. In this equation, $\eta^{\mathcal{M}}$ is the model error and $\boldsymbol{\varepsilon}^{o}$ is the observational error, which includes the (representativity) error relative to the observation operator. If they are small enough (in particular their systematic parts), all these errors eventually grow or decrease depending on the amplitudes of their components on the unstable directions.

Assimilating the same observations by the same scheme in the perturbed and in the control trajectories, the perturbation equation for the system subject to observational forcing, Eq. (4), is:

$\delta \mathbf{x}_{k+1}^{a}=(\mathbf{I}-\mathbf{K H}) \mathbf{M} \delta \mathbf{x}_{k}^{a}$ 
By effect of the matrix (I-KH), and depending on its properties, the analysis has, generally speaking, a stabilizing effect. In some circumstances, it may happen that the unstable components are amplified by effect of the assimilation, but assimilation schemes in general, and particularly the present formulation, are designed to reduce the rapidly amplifying components of the error. As a consequence, the number of independent unstable directions relative to Eq. (6) and their associated growth rates are smaller than those relative to Eq. (2). In practice, in an intermittent assimilation system, during the forecast step the perturbations growth is dominated by the free system instabilities, and, at the analysis step, some unstable components are reduced by the assimilation of observations. Therefore some of the unstable components present in the forecast error are absent, or at least reduced, in the analysis error. After the analysis step, however, some unstable components, either because they have not been completely eliminated, or, in the worst cases, because they have been introduced by the analysis, are still present and will grow during the next forecast step.

In order to account for the observational forcing in the estimate of the instabilities that grow along the assimilation cycle, we adopted a modified form of breeding, that is referred to as Breeding on the Data Assimilation System (BDAS), based on Eq. (6) rather than Eq. (2). As in classic breeding, the control parameters are the normalization amplitude and frequency, and the length of the breeding period. Details on the choice of these parameters are given in Sects. 4 and 5.

\subsection{Assimilation in the Unstable Subspace (AUS)}

The forecast error can be seen as the sum of two components:

$\eta^{f}=\mathbf{E} \boldsymbol{\gamma}+\boldsymbol{\xi}$

where $\mathbf{E} \boldsymbol{\gamma}$ is the forecast error component on the subspace spanned by $N$ estimated unstable directions, stored as columns in a matrix $\mathbf{E}$ of dimension $(I, N)$, and $\xi$ is the forecast error component on the complementary subspace. $I$, the number of rows in $\mathbf{E}$, is the state dimension and the vector $\gamma$ represents the forecast error component in the unstable basis. This implies:

$\mathbf{P}^{f}=\mathbf{E} \boldsymbol{\Gamma} \mathbf{E}^{\mathrm{T}}+\mathbf{E}\left\langle\gamma \xi^{\mathrm{T}}\right\rangle+\left\langle\xi \gamma^{\mathrm{T}}\right\rangle \mathbf{E}^{\mathrm{T}}+\left\langle\xi \xi^{\mathrm{T}}\right\rangle$

where $\mathbf{P}^{f} \equiv\left\langle\boldsymbol{\eta}^{f}\left(\boldsymbol{\eta}^{f}\right)^{\mathrm{T}}\right\rangle$ is the forecast error covariance and $\Gamma \equiv\left\langle\gamma \gamma^{T}\right\rangle$ is the forecast error covariance in the unstable subspace. Because unstable directions grow, they dominate the forecast error. If terms containing $\xi$ are neglected in Eq. (8):

$\mathbf{P}^{f} \simeq \mathbf{P}_{E E}^{f}=\mathbf{E} \boldsymbol{\Gamma} \mathbf{E}^{\mathrm{T}}$

then, for a given observation operator $H$, the minimum error variance analysis corresponding to the approximation (9) is:

$$
\begin{aligned}
& \mathbf{x}^{a}= \mathbf{x}^{f}+\mathbf{E} \boldsymbol{\Gamma}(\mathbf{H E})^{\mathrm{T}}\left[(\mathbf{H E}) \boldsymbol{\Gamma}(\mathbf{H E})^{\mathrm{T}}+\mathbf{R}\right]^{-1} \\
& \cdot\left[\mathbf{y}^{o}-H\left(\mathbf{x}^{f}\right)\right]
\end{aligned}
$$

where $\mathbf{R}$ is the observational error covariance matrix. In this way, the analysis increment is confined to the $N$-dimensional subspace spanned by the columns of $\mathbf{E}$ : for this reason the present scheme is referred to as Assimilation in the Unstable Subspace (AUS). A useful expression, equivalent to Eq. (10), is:

$$
\begin{aligned}
\mathbf{x}^{a}= & \mathbf{x}^{f}+\mathbf{E}\left[\boldsymbol{\Gamma}^{-1}+(\mathbf{H E})^{\mathrm{T}} \mathbf{R}^{-1}(\mathbf{H E})\right]^{-1}(\mathbf{H E})^{\mathrm{T}} \mathbf{R}^{-1} \\
& \cdot\left[\mathbf{y}^{o}-H\left(\mathbf{x}^{f}\right)\right]
\end{aligned}
$$

In the general expression of the Kalman analysis, that can be compared with Eq. (10), the "complete" $\mathbf{P}^{f}$ appears, instead:

$\mathbf{x}^{a}=\mathbf{x}^{f}+\mathbf{P}^{f} \mathbf{H}^{\mathrm{T}}\left[\mathbf{H} \mathbf{P}^{f} \mathbf{H}^{\mathrm{T}}+\mathbf{R}\right]^{-1}\left[\mathbf{y}^{o}-H\left(\mathbf{x}^{f}\right)\right]$

where the gain matrix, as defined in Eq. (3), is the Kalman gain. In this expression, if $M$ is the number of observations, the analysis increment appears confined to the $M$ dimensional subspace spanned by the "representers" (Bennett, 1992, 2002; Uboldi and Kamachi, 2000), that is to say the columns of the $(I, M)$ matrix $\mathbf{P}^{f} \mathbf{H}^{\mathrm{T}}$. In a stationary analysis scheme, a fixed "background" error covariance matrix, $\mathbf{B}$, is used instead of $\mathbf{P}^{f}$ : in such schemes the $M$ columns of the matrix $\mathbf{B H}^{\mathrm{T}}$ span the subspace where the analysis increment is confined. In the present scheme, if $M \geq N$, the $M$ observations are used, in Eq. (10), in a least-square sense, to compute an analysis increment that is constrained into the selected $N$-dimensional subspace.

The neglected components of $\mathbf{P}^{f}$ in Eq. (8) are covariances between (and with) directions belonging to the linear complement of the subspace spanned by the columns of $\mathbf{E}$. If the gain matrix is the "complete" Kalman gain, as in Eq. (12), then the Kalman filter equations provide the technique for updating the "complete" forecast error covariance to that of the analysis (and, then, of the successive forecast). If, however, the gain matrix is computed in a subspace, by using an approximation similar to Eq. (9) for the forecast error covariance, as it is also done in EnKFs and in other "suboptimal" filters (differences being in how the subspace is estimated), then the analysis has the effect of reducing the error components in that subspace. This means that, on the one hand, the amplitude of the error is reduced, but, on the other hand, that the relative amplitude of its components on the complementary subspace is increased. In other words, the directions (the ensemble) just used for estimating $\mathbf{P}^{f}$ and $\mathbf{K}$ are less valuable after the analysis step: the error reduction is obtained by "spending" part of the information previously available on the error covariance. This is the reason why a "refresh" procedure is introduced here: details are given, with reference to the implementation choices, in Sect. 4. This is also the reason why, in EnKFs, it is appropriate to implement some sort of re-sampling or ensemble re-initialization (Whitaker and Hamill, 2002). Evensen (2003) reviewed some of the techniques that have been implemented to this purpose in EnKFs. Corazza et al. (2002) showed that refreshing bred vectors improved substantially their efficacy. 
The experiments presented in this work are made in a perfect model and perfect observation setting, that allows to highlight the mechanism discussed above for explaining the need of a refreshing procedure. In case a small model error is introduced at each time step during the model integration, it is subject to the perturbation dynamics. Then it also progressively acquires the structure of the unstable vectors as the time integration proceeds. The resulting effect on the forecast error is a larger uncertainty on its components along the unstable directions. These amplitudes can be measured by the observations if they are appropriately located (Sect. 2.3). The "yet unstructured" components of the model error increase the neglected part of the forecast error, $\xi$. The importance of the refresh procedure seems then enhanced by the presence of model error. These concepts appear worthy of investigation in future work.

\subsection{Unstable directions and observations}

For each estimated unstable direction one or more observations can be used to eliminate or reduce the error component along that direction. In order to show how this is carried out, we consider the case of a single unstable direction $\mathbf{e}$, $M \geq N=1$. The analysis is, from Eq. (11):

$\mathbf{x}^{a}=\mathbf{x}^{f}+\mathbf{e} \frac{(\mathbf{H e})^{\mathrm{T}} \mathbf{R}^{-1}\left[\mathbf{y}^{o}-H\left(\mathbf{x}^{f}\right)\right]}{\gamma^{-2}+(\mathbf{H e})^{\mathrm{T}} \mathbf{R}^{-1} \mathbf{H e}}$

where the $(N, N)$ matrix $\boldsymbol{\Gamma}$ is reduced to the scalar $\gamma^{2}$. It can be seen that the direction of analysis increment is that of the single unstable direction, the vector $\mathbf{e}$, while the amplitude of the correction is estimated by means of the $M$ observations, weighted with their uncertainties.

In the case of perfect observations it becomes:

$\mathbf{x}^{a}=\mathbf{x}^{f}+\mathbf{e} \frac{(\mathbf{H e})^{\mathrm{T}} \boldsymbol{\Sigma}^{-1}\left[\mathbf{y}^{o}-H\left(\mathbf{x}^{f}\right)\right]}{(\mathbf{H e})^{\mathrm{T}} \boldsymbol{\Sigma}^{-1} \mathbf{H e}}$

where the matrix $\boldsymbol{\Sigma}$ may contain the co-variabilities of the observed variables (their physical dimensions can be different from each other). Equation (14) minimizes the $\boldsymbol{\Sigma}^{-1}$ distance between the innovation and the "observed" component, $\mathbf{H}\left(\mathbf{x}^{a}-\mathbf{x}^{f}\right)$, of the analysis increment, strongly constrained in the e direction.

If the observed variables are physically homogeneous and uncorrelated, with the same error variance $\sigma^{2}$, then Eq. (13) becomes, after small rearrangements:

$\mathbf{x}^{a}=\mathbf{x}^{f}+\mathbf{e} \frac{(\mathbf{H e})^{\mathrm{T}}\left[\mathbf{y}^{o}-H\left(\mathbf{x}^{f}\right)\right]}{(\mathbf{H e})^{\mathrm{T}} \mathbf{H e}} \frac{\gamma^{2}(\mathbf{H e})^{\mathrm{T}} \mathbf{H e}}{\sigma^{2}+\gamma^{2}(\mathbf{H e})^{\mathrm{T}} \mathbf{H e}}$

When a single observation is used with a single unstable direction, $M=N=1, y^{o}$ and $\mathbf{H e}$ are scalars and:

$\mathbf{x}^{a}=\mathbf{x}^{f}+\mathbf{e} \frac{y^{o}-H\left(\mathbf{x}^{f}\right)}{\mathbf{H e}} \frac{\gamma^{2}(\mathbf{H e})^{2}}{\sigma^{2}+\gamma^{2}(\mathbf{H e})^{2}}$

In Eqs. (15) and (16), the last ratio on the right-hand side accounts for observational errors, and reduces to 1 when observations are considered perfect with respect to the forecast state. By considering Eq. (16), it can be seen that the amplitude of the correction (in the direction of the single unstable direction e) fits the single observation, exactly if this is perfect. In this work, only experiments with perfect observations are presented; for results obtained with noisy observations in the context of a quasi-geostrophic model of the atmosphere, and an estimate of $\gamma^{2}$, see CTU06.

In the rightmost denominator appearing in Eq. (15), the observational error variance becomes less important when many observations are available and each of them detects a relevant component of the vector $\mathbf{e}$, so that $(\mathbf{H e})^{\mathrm{T}} \mathbf{H e}$ is large. Therefore the perfect observation case can be considered representative of a situation where there are many noisy observations available at the appropriate locations. In general, referring to Eq. (11), the observation locations and variables (as represented by the components of $\mathbf{H}$ ) are most valuable when a measure (for example the trace) of the matrix $(\mathbf{H E})^{\mathrm{T}} \mathbf{R}^{-1}(\mathbf{H E})$ is large with respect to that of $\boldsymbol{\Gamma}^{-1}$. This criterion can be used as a guideline for choosing observation locations (and variables) when it is possible to take "adaptive" or "targeted" observations. Other criteria were discussed by Berliner et al. (1999). In practice, in the experiments presented here, we put a single perfect observation in the maximum of each estimated unstable "structure" (see Sect. 5 for details).

As described in Sect. 4, when only a fixed network of observations is available, it can be used to detect the unstable structures if these happen to be located, at observation time, in geographical regions where standard observations are available. If the unstable structures are not detected by the standard observation network, they still may migrate in an observed region, before errors become too large, and be detected at a later observation time. In any case, our ability to control the system depends on the number of unstable structures present and on their growth rate, in relation to the frequency and distribution of observations by which we can detect and eliminate them.

\section{The ocean system and the standard assimilation method}

The primitive equation ocean model MICOM (Bleck, 1978) is set up here in a simplified adiabatic configuration: a flat bottom basin, with a $180 \times 140$ horizontal grid and 4 isopycnal layers, with constant surface wind forcing. Variables are the layer thicknesses (or, alternatively, the layer interface elevations) and the zonal and meridional components of velocity, defined on an Arakawa $\mathrm{C}$ scattered grid. The number of independent components in the state vector is 301120 . The layer densities are $1024.590 \mathrm{~kg} \mathrm{~m}^{-3}$, $1026.520 \mathrm{~kg} \mathrm{~m}^{-3}, 1028.187 \mathrm{~kg} \mathrm{~m}^{-3}$ and $1030.130 \mathrm{~kg} \mathrm{~m}^{-3}$. The average depths of the internal interfaces between layers are $440 \mathrm{~m}, 1048 \mathrm{~m}$ and $2026 \mathrm{~m}$, while the bottom depth is fixed at $5000 \mathrm{~m}$. The horizontal resolution is approximately $20 \mathrm{~km}$, with latitudes ranging from $27.12 \mathrm{~N}$ to $52.12 \mathrm{~N}$, and a longitude interval of 42 degrees. 
The typical dynamic situation is that of a double gyre, cyclonic in the northern half of the domain, anticyclonic in the southern half, with an eastward jet detaching from the western boundary and a wide recirculation region in the eastern part of the domain. The eastward jet is characterized by sharp meanders and energetic eddies; moreover, large velocity gradients appear in regions that are close to the western boundary. The leading Lyapunov exponent of the (freely evolving) system has been approximately estimated (from a 6-year integration) and corresponds to a doubling time of about 19 days.

Experiments are made with perfect model conditions (identical twins): a 6-year model trajectory represents the truth, from which perfect observations are taken. The fixed observational network consists of "satellite" sea surface height, observed every 10 days on the whole domain from the true trajectory. A standard assimilation scheme is also available, the Cooper and Haines (1996) scheme, hereafter $\mathrm{CH}$, based on conservation of linear potential vorticity, and characterized by a homogeneous vertical shift of isopycnals, with no change in the bottom pressure. This scheme, that consists in a vertical displacement of the internal layer interfaces and geostrophic adjustment of velocity, has the advantage of being particularly simple to implement with MICOM. The most important limitations of the scheme are that it is stationary (i.e. assumes constant forecast error covariance) and that it accounts only for one vertical "mode". Other stationary schemes, in which more vertical modes are considered in the estimation of the background error covariance (De Mey and Benkiran, 2002, for example) may have a superior performance; however the $\mathrm{CH}$ mode, at least, accounts for a vertical displacement of the pycnocline without degrading its signal, as it may happen when using statistics based on climatology.

As it was shown in UTC05, the standard $\mathrm{CH}$ assimilation scheme is able to reduce an initially large error (difference between two randomly chosen states) to values oscillating between 0.3 and 0.4 of the natural variability. The error reaches the same range of values in one year even when starting from a small value (initially rescaled to 0.1 of natural variability) as the standard $\mathrm{CH}$ scheme is unable to maintain the initially small error.

Error structures which are well corrected by the $\mathrm{CH}$ scheme are characterized by an error in the depth of the pycnocline, which can be reduced by modifying the thicknesses of the first and bottom layers only, so that they compensate each other without changing the bottom pressure. In UTC05, it was shown that errors having vertical structure different from the $\mathrm{CH}$ mode appear frequently. Errors present in the forecast are mostly located either in the region of the eastward jet (see e.g. Fig 4), which detaches from the western boundary between latitudes $39 \mathrm{~N}$ and $42 \mathrm{~N}$, or within the two western boundary currents associated with the northern cyclonic and southern anticyclonic gyres (Figs. 6 and 7 of UTC05). The $\mathrm{CH}$ corrections are mostly located in the eastward jet region, while other errors are insufficiently corrected, to the point that the analysis error is even larger than the forecast error in the northwestern part of the domain. These results support the need for more realistic, flow-dependent, dynamically consistent vertical covariances in the assimilation of surface height observations. Brasseur et al. (1999) used the same model MICOM, in a configuration that appears similar to that used in the present study, but with a much smaller basin. They showed that the dynamically evolving SEEK (Singular Evolutive Extended Kalman) filter, even with few ensemble members, quickly approached small values of forecast errors, as estimated by the 10-day SSH innovation.

\section{Assimilation of standard observations}

In UTC05, the BDAS-AUS assimilation has been applied with the standard observational network described above. There, it is shown that at least some of the unstable components present in the forecast error are detected and eliminated, by taking advantage of the fixed SSH observations only, assimilated in the estimated unstable subspace. Referring the reader to UTC05 for details, we briefly recall here how BDAS and AUS are implemented in this configuration.

A CH analysis scheme starting from a climatological initial forecast state reaches in a year its typical error values, between 0.3 and 0.4 of natural variability. At this point (day 360 ), perturbations are initially introduced and bred for 2 months, during which the only assimilation running is still $\mathrm{CH}$. At each SSH assimilation time (every 10 days), after the analysis step, a set of 6 independent (total-energyorthogonal) perturbations are introduced; initial perturbations are built randomly as differences between model states. Before forecast integration, all perturbations, old and new, are rescaled to 0.1 of natural variability (total energy norm). At the end of the initial 2 month period (day 420), 36 perturbed trajectories are being integrated. At day 420, the new assimilation system begins to act. Each of the 6 "current" bred perturbations, the oldest set, is searched for local maxima and minima structures, starting from the largest (in absolute value).

The need to isolate local maxima stands from the fact that bred vectors are (approximately) combinations of Lyapunov vectors (Trevisan and Pancotti, 1998) so that the forecast error may have opposite correlations with different local structures appearing in the same bred vector.

At analysis time the following four steps are taken (details in UTC05).

1. Regionalization, obtained by point-by-point multiplication of the bred vector with an elliptically shaped Gaussian, whose position, shape, orientation and width are chosen (by least-square minimization) in order to isolate, without deformation, the regional structure of the bred vector surrounding a local maximum or minimum, from other structures or signals present in the same bred vector.

2. Selection, obtained by comparing each structure (obtained by regionalization) with the innovation (sea sur- 
face elevation field) and retaining only those that have a regional correlation larger than 0.8 in absolute value. Of course, this step had to be modified for the targeted vertical profiles, as explained in Sect. 5.

3. Assimilation. The selected structures are used one by one in a sequence. The reason for this choice is to rule out the possibility that, when the same local structure appears in two different bred modes, anomalously large corrections may be introduced in the (non-significant) direction of the local difference between the two vectors: this may be particularly dangerous with regard to the unobserved components. The analysis, obtained with a single structure by means of Eq. (15) after setting $\sigma^{2}=0$, is used as background field in the analysis with the next selected structure. After the last structure has been used, the innovation has been significantly reduced, but still it is different from zero, thus a standard $\mathrm{CH}$ analysis is performed. The assimilation of targeted vertical profiles, performed at times when SSH observations are not available, is discussed in Sect. 5.

4. Refresh. The reasons for introducing a refresh procedure are explained in Sect. 2.2. However, we point out that the current bred vectors, from which the regionalized unstable structures have been extracted and used in the assimilation, still carry useful information, so we do not discard the current set, in contrast with what has been done with less complex systems (TU04, CTU06). The following procedure has been implemented as a trade-off between keeping and discarding the bred vectors altogether. After the assimilation, one (at turns) of the current, just used, bred vectors is point-by-point added to all the other 5 vectors of the same set. One new random perturbation is inserted in its place, so that a new set of 6 vectors is obtained. These vectors are totalenergy-orthogonalized only at this point, before starting to undergo a new 60-day breeding cycle.

Results (from a longer run with respect to UTC05) are included, together with targeted observations results, in Fig. 1 and are commented in Sect. 5.2.1.

\section{Assimilation of targeted observations}

The bred vectors obtained by BDAS, subject to renormalization and refresh at SSH assimilation times (as described in Sect. 4), being differences between the perturbed states and the control state, can be computed at any time, in particular at times that are intermediate between two successive $\mathrm{SSH}$ assimilation times. At these intermediate times they are used to locate and assimilate adaptive observations.

\subsection{Implementation}

In the experiment shown here, the unstable structures are estimated at $+2.5 \mathrm{~d},+5.0 \mathrm{~d}$, and $+7.5 \mathrm{~d}$ with respect to the last SSH assimilation (while the next SSH assimilation is at
$+10 \mathrm{~d})$. At each of these intermediate times, 3 adaptive observations are taken. Each observation is designed to simulate a vertical profile of temperature and salinity, from which the vertical density profile can be inferred. When a real vertical profile is taken, however, it is difficult to obtain a precise measure of the sea surface height (with respect to a reference depth), particularly in comparison with what can be obtained by satellite measurements. For this reason, in our simulated profiles, we choose not to use the information on the sea surface elevation, but only that on the elevation of the 3 internal layer interfaces, i.e. that between layers $1-2$, layers $2-3$, and layers 3-4. These three values are used in the "selection" step, described below, while only one of the three scalar values is actually assimilated.

In fact, targeting is obtained by choosing, among the current set of 6 forced bred vectors, 3 local maxima or minima in the interface elevation fields: their positions are identified by the 2 horizontal discrete coordinates and by the vertical one. We anticipate here that the vertical position of the maximum of the structure is important. The 3 structures are chosen with the condition that they are horizontally well separated.

The regionalization procedure is the same as described in Sect. 4.

The selection procedure described for SSH observation in Sect. 4 has been implemented for the "vertical profile" observations with the obvious modification that the "observed" component of the structure, $\mathbf{H e}$, and the innovation, $\mathbf{d}$, consist of the three layer interface elevations. Observations are retained for assimilation only if $\mathbf{H e}$ and $\mathbf{d}$ have a correlation higher than 0.6, while a smaller value determines rejection. The assimilation of the 3 observations, one scalar observation for each profile (the elevation of the targeted layer interface), is done in a sequence: the analysis field obtained after the assimilation of one profile is used as background field for the next one. This choice is made, rather than assimilating the 3 observations together, for the same reasons explained in the description of the "assimilation" procedure step in Sect. 4.

Each observation is assimilated by means of Eq. (16), with $\sigma^{2}=0$ : it is clear that the observation is only used to estimate the amplitude of the correction, while its 3-D structure, in all the state variables, is that of (the regionalized structure of) the bred vector, stored in the vector $\boldsymbol{e}$. This estimate is obtained, in the present case, by a single perfect observation. In a realistic case, where observations are affected by errors, it might be necessary to assimilate, by means of Eq. (15), more observations: either more components from the same profile, or possibly more profiles (eventually taken by the same ship), located in the region where the unstable structure is active.

As an example, Fig. 6a shows the analysis increment computed from three bred vectors shown in Fig. 5.

\subsection{Results}

\subsubsection{Global error}

A scalar measure of the error on the whole state, that is to say on all layers and variables, including velocity components, 
(a)

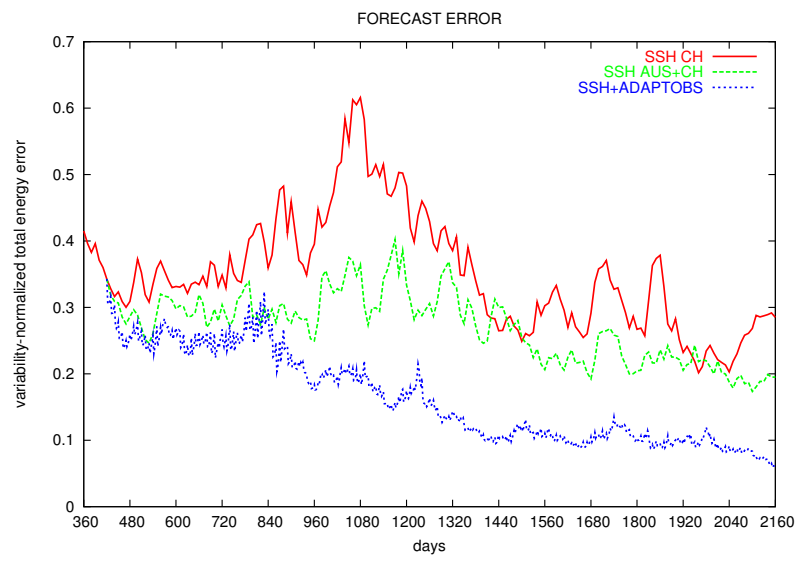

(b)

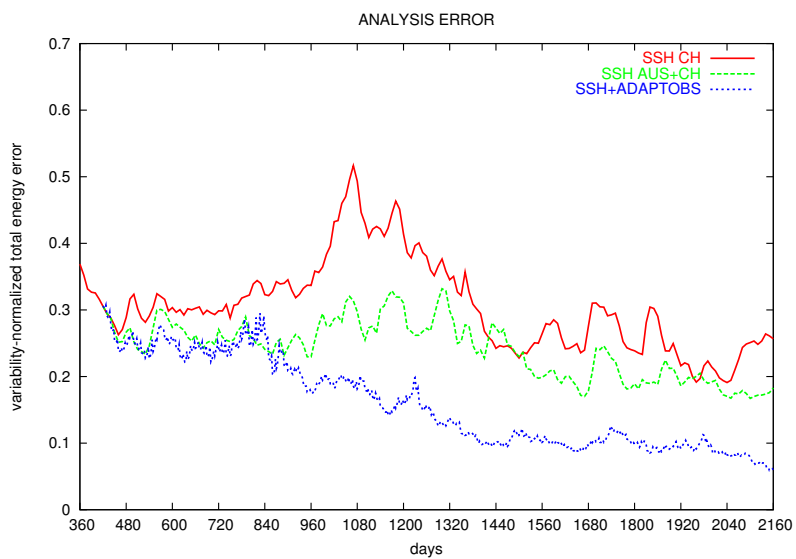

Fig. 1. Square root of total energy error (normalized with natural variability) as a function of time: (a) forecast error; (b) analysis error. "SSH CH": standard CH assimilation of SSH only; "SSH AUS+CH": assimilation of SSH observations only by AUS and CH, as described in Sect. 5; "SSH+ADAPTOBS": AUS+CH assimilation of SSH and supplementary adaptive observations, as described in Sect. 5.1.

is obtained by a total energy norm (normalized with natural variability computed from a 6-year model trajectory). The global error is defined here as the square root of its total energy, which is a quadratic function of the errors on the state variables (interface elevation and velocity components). The forecast error is shown in Fig. 1a, and the analysis error in Fig. 1b, for three experiments over a 5-year period, from day 360 (after a year in which only the standard $\mathrm{CH}$ assimilation of SSH is active) to day 2160. In the first experiment, the standard $\mathrm{CH}$ analysis of SSH is performed every 10 days. It can be seen that a high variability of the error is present; in particular there is a period, around day 1080, in which errors are as high as 0.6 of natural variability. In the second experiment, only the SSH observations are used with the BDASAUS assimilation described in Sect. 4 and in UTC05. As discussed in UTC05, this scheme is successful in controlling the worst error peaks and other oscillations. The third experiment is the one with the adaptive observations located and assimilated as described in Sect. 5.1. As can be seen, after an initial period of about one year, in which the errors are only a little smaller than in the experiment without adaptive observations, errors start to systematically decrease and during the last 28 months both the forecast error and analysis error reach values as low as 0.1 of the natural variability.

It is worth noticing that errors start to decrease in a period when the SSH standard assimilation alone fails to keep the error within low bounds. In this period (from day 840 to day 1320) the flow is characterized by the presence of particularly strong and persistent meanders in the eastward jet; unstable structures present in the bred vectors are many, and concentrated in the meander regions. A dynamically consistent assimilation is particularly important in this situation. Control of instabilities is partially obtained by exploiting the surface height information, but it is fully achieved by making use of the adaptive observations.
Another important comment is that the difference between the forecast error curve (Fig. 1a) and the analysis error curve (Fig. 1b) is smaller for the BDAS-AUS dynamically consistent assimilation of SSH with respect to what happens with the stationary $\mathrm{CH}$ assimilation, and it is confined to even smaller values for the adaptive observation experiment. This is an indication of a slower error growth, during the forecast step, achieved by a successful control of the instabilities.

\subsubsection{Space distribution of adaptive observations}

The number of adaptive observations located at each grid point and at the different layer interfaces indicates how the instabilities are structured horizontally and at the different depths.

Figure 2 a shows the horizontal distribution of 359 observations of the elevation of the interface between layers 1 and 2: these observations are mostly located in the eastward jet region. The corresponding unstable structures are connected with fronts and gradients that characterize the eastward jet, its meanders and eddies.

Most of the observations, 736, are located at the deepest interface, between layers 3 and 4, and their horizontal positions are shown in Fig. 2b. These observations are mostly located near the western boundary. The corresponding, "deep", unstable structures are connected with the strong velocity gradients that appear in this area where western boundary currents intensify in the proximity of the vertical "continental shelf", before detaching and giving origin to the eastward jet. Only 34 targeted observations are located at the interface between layers 2 and 3: their distribution, not shown, is similar to that of the deepest observations, Fig. 2 b.

There is a very close correspondence between the horizontal and vertical distribution of the adaptive observations and the average forecast error fields, as can be seen by comparing Fig. 2 and Fig. 3. This correspondence further corroborates the conclusion that the forced bred modes, by means 
(a)

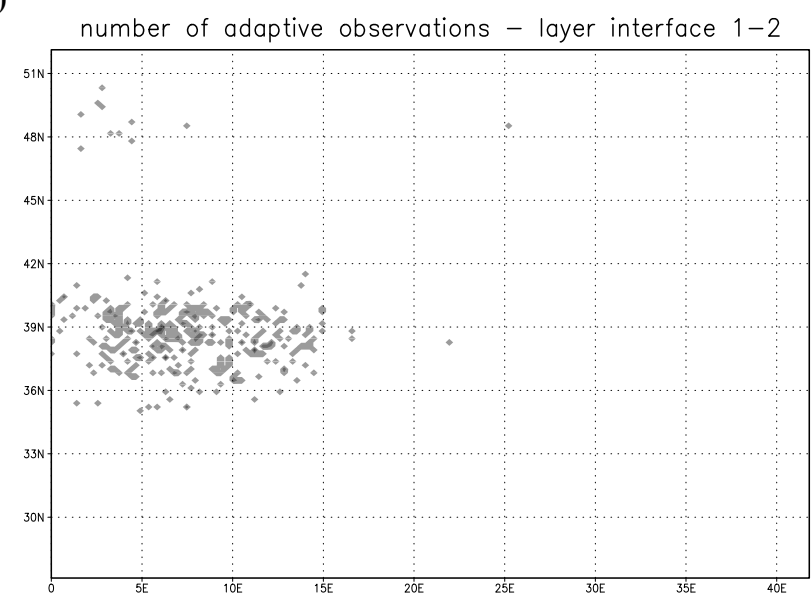

(b)

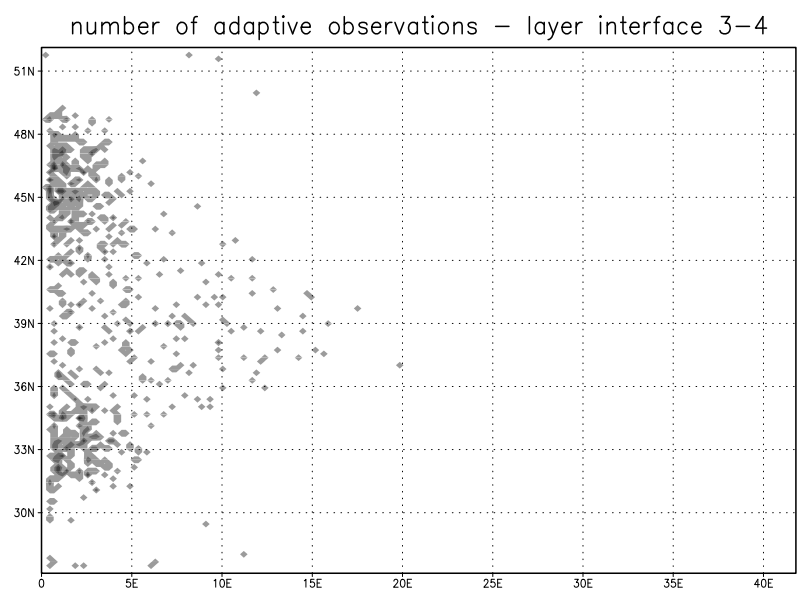

Fig. 2. Number of adaptive observations per gridpoint: (a) interface between layers 1 and 2; (b) interface between layers 3 and 4 . Contouring values are set to: $0.1,1.1,2.1,3.1$.

(a)

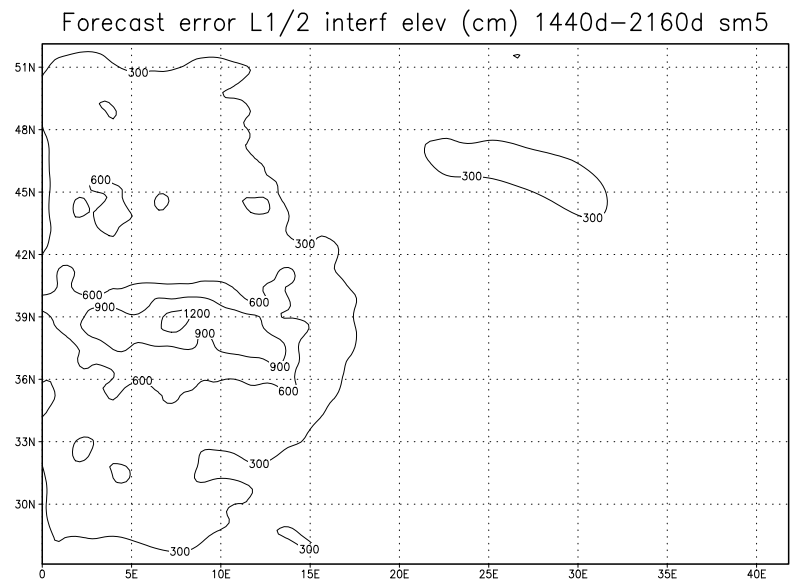

(b)

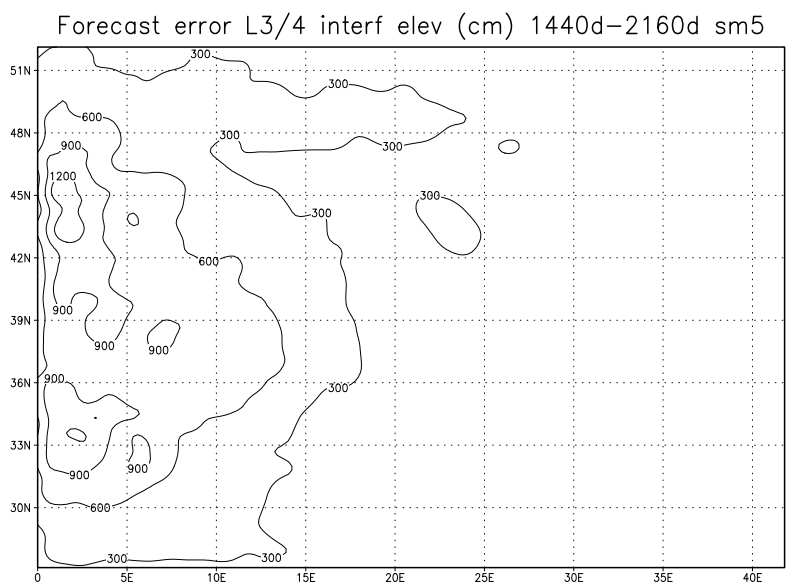

Fig. 3. Forecast error at SSH assimilation time, averaged in time from day 1440 to day 2160 (the 2-year period in which the errors are stabilized at about 0.1 of natural variability): (a) elevation of interface between layers 1 and 2; (b) elevation of interface between layers 3 and 4. Both fields have been slightly smoothed out.

of which the adaptive observations were located, capture the relevant part of the forecast error.

\subsubsection{Errors on variables and layers}

For each variable, on each layer, the Root-Mean-Square (RMS) errors have been computed, and their time evolution examined. They are not shown here, however all of them reflect the characters of the global error curves shown in Fig. 1: the adaptive observation experiment has a drastic and stable reduction of errors, starting from about day 1320 , and smaller differences between forecast and analysis errors. With regard to errors in the velocity fields at the different layers, for the standard $\mathrm{CH}$ assimilation of SSH the difference between the forecast error and the analysis error is much more pronounced for layers 1 and 2 than for the deeper layers 3 and
4. This is partly true also in the experiment with the BDASAUS dynamical assimilation of SSH, while this difference between surface and bottom layer velocities does not appear at all in the adaptive observations experiment, in particular after the errors start to decrease. Differences between forecast and analysis errors at the surface are reduced by means of adaptive observations that (Sect. 5.2.2) are mostly located in the deeper layers. We conclude, from a "forecaster" point of view, that the prediction of currents in layers that are close to the surface is sensitive to errors present in the analysis field in deeper layers. From an "oceanographer" point of view, this is an indication of how the dynamical activity in the upper ocean is sensitive to perturbations of the mass and velocity fields in deeper layers. 


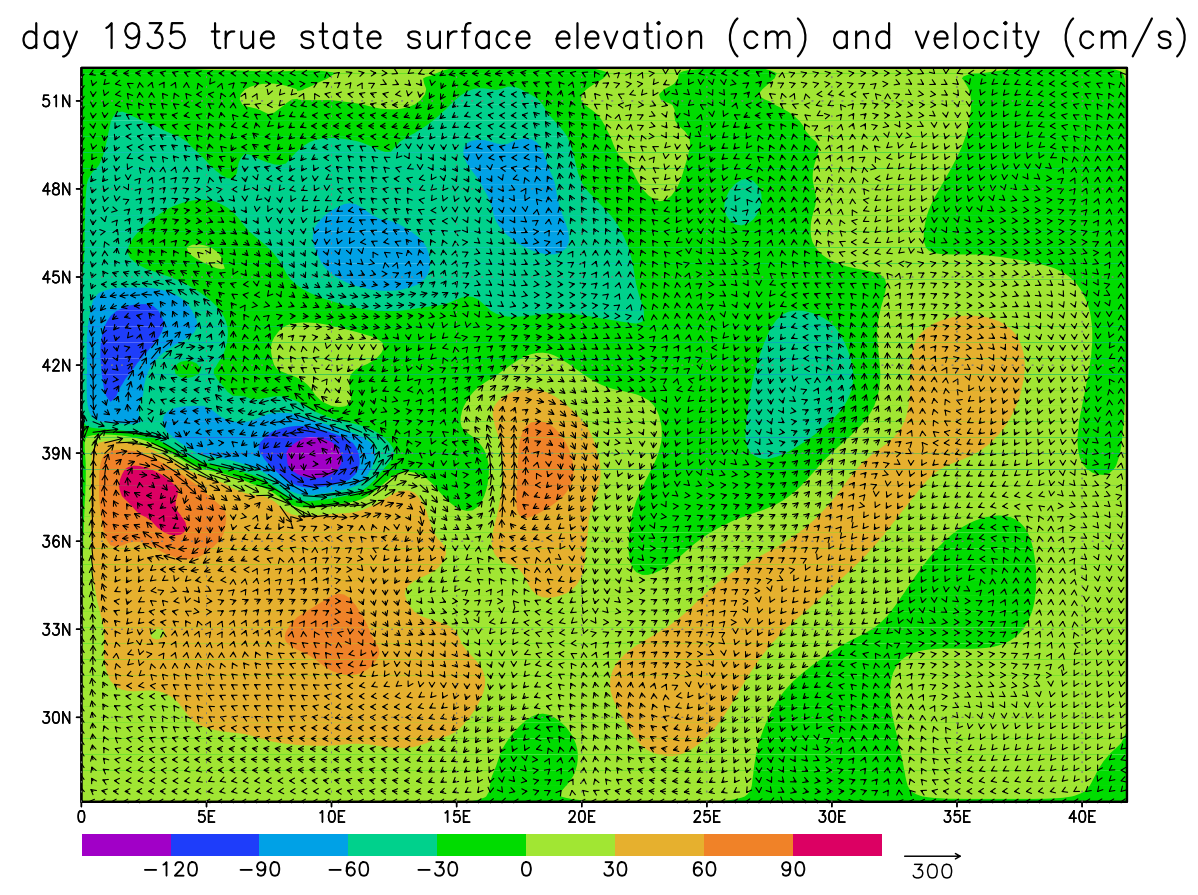

Fig. 4. True state at day 1935. SSH field (colour shading) and layer 1 velocity (arrows).

\subsubsection{Bred vectors, analysis increment and forecast error: adaptive observations at day 1935}

In order to show how the analysis procedure works, it is interesting to examine one case in detail. To get an idea of how the flow is at a particular instant (day 1935), Fig. 4 shows the "true" state sea surface height field, with, superimposed, the velocity vectors. Three adaptive observations have been taken and all have been assimilated in this case.

Figure 5 shows, in its panels (a), (c) and (e) the bred vectors and, in its panels (b), (d) and (f), the corresponding local structures that have been extracted, following the procedure described in Sect. 5.1. The field shown in each plot is the interface elevation where the maximum is located in the vertical: in all the three cases the targeted interface is that between layer 3 and 4, so these are "deep" structures. The target positions for the observations are the maxima, marked with black dots. All three assimilated values are elevations of the interface between layers 3 and 4 , corresponding to the vertical position of the structures' maxima.

Figure 6a shows contour lines of the analysis increment in this field, superimposed to the forecast error for the same field, shown as colour shading. As can be seen, the structures used to compute the analysis increment are very well correlated with structures that are present in the forecast error field. This is true in particular for the two observations located at approximately $5 \mathrm{E}, 38 \mathrm{~N}$ and $4 \mathrm{E}, 43 \mathrm{~N}$. The structure located at $2 \mathrm{E}, 47 \mathrm{~N}$ has a very small amplitude (measured by the observation) in the forecast error field, and the analysis increment is correspondingly small. In the forecast error fields there exist other structures that could not be detected at this time by using three observations only. However, in this case, these other structures have been controlled by means of other observations, taken at a later time; in fact the global error curve in Fig. 1 continues to decrease in the period immediately after day 1935 .

It is particularly important to remark that the vertical structure of the analysis increment is correct. Figure $6 \mathrm{~b}$ shows the analysis increment relative to the same observations, together with the forecast error, but for the sea surface elevation. The horizontal position of the (deeply located) targeted observations is marked in this figure too, to illustrate how these structures also change in the vertical direction: for example, the structure located at about $5 \mathrm{E}, 38 \mathrm{~N}$ is evidently tilted. However, the correlation between the analysis increment and the forecast error is high even at depths that are different from where the observations are located.

Corazza et al. (2003) showed a similar comparison between the background error and the free (i.e. not forced by the assimilation of observations) bred modes, that were used (Corazza et al., 2002) to build a flow-dependent background error covariance matrix.

\subsubsection{Analysis increment and forecast error: assimilation} of surface height at day 1940

It is also instructive to illustrate in detail how the BDAS-AUS dynamically consistent assimilation works with the standard SSH observations. The case of day 1940 is considered here.

Figure 7a shows, as contour lines, the analysis increment computed by using the structures present in the bred vectors, and extracted by regionalization, in comparison with the forecast error on the same field. 
(a)

day 1935; bred vector N. 1; layers 3-4 interface elevation

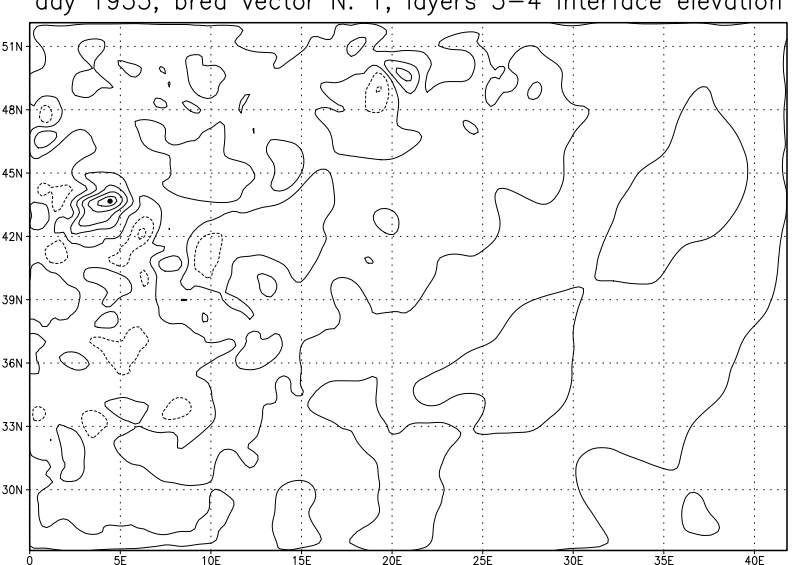

(c)

day 1935; bred vector N. 5; layers 3-4 interface elevation

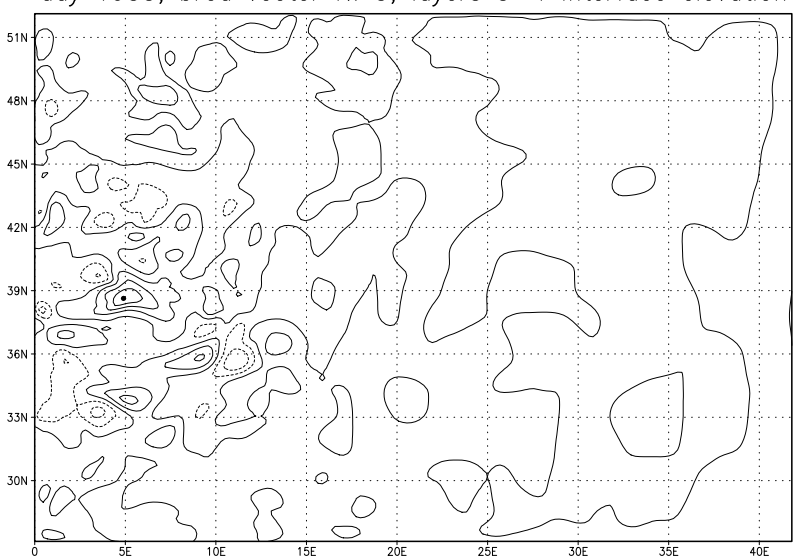

(e)

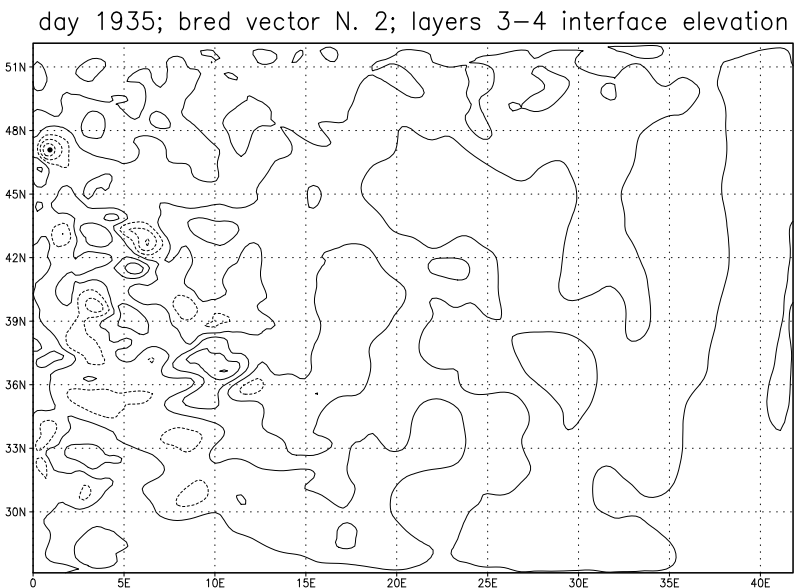

(b)

day 1935; regionalized bred vector N.1; L 3-4 interface elevation

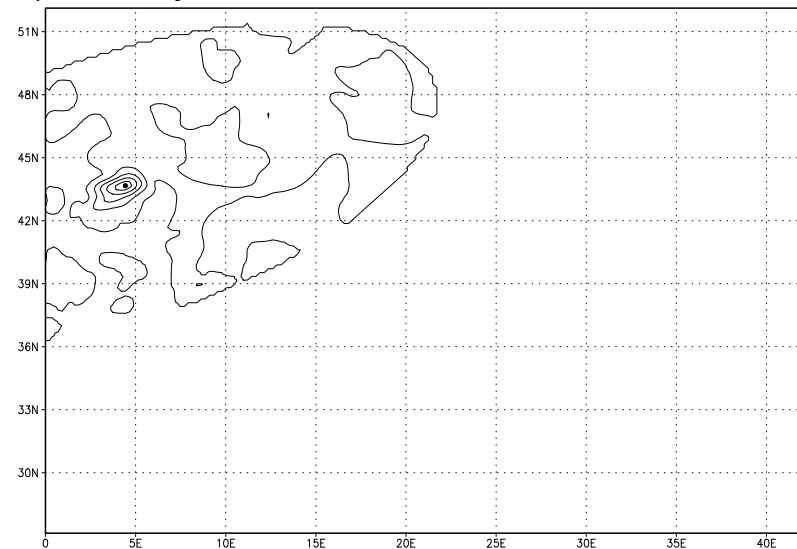

(d)

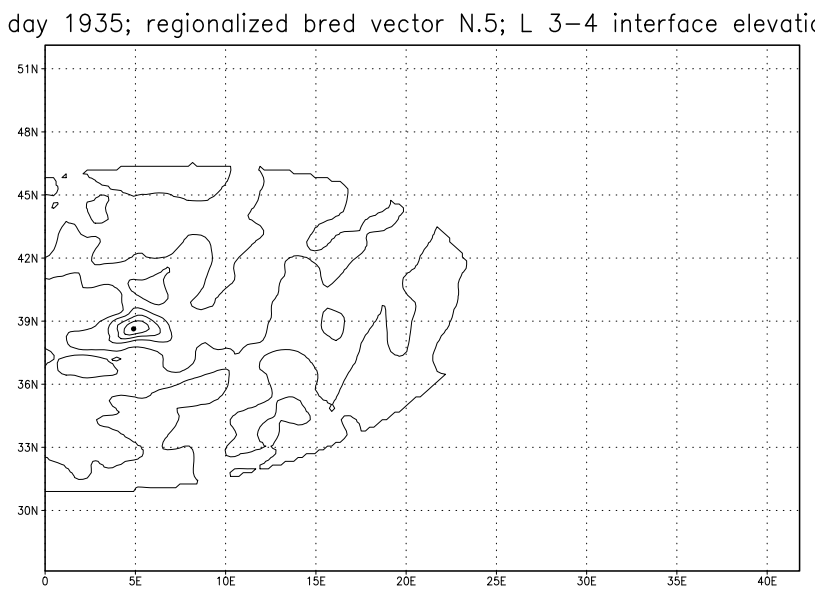

(f)

day 1935; regionalized bred vector N.2; L 3-4 interface elevation

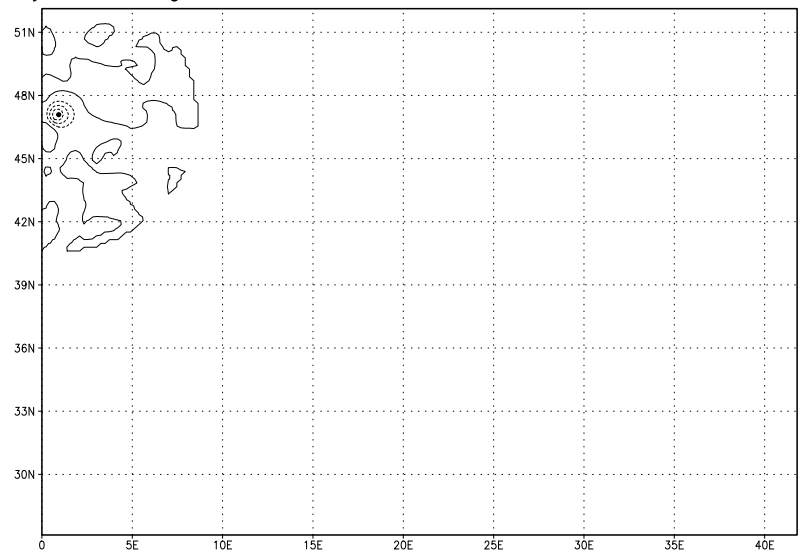

Fig. 5. Day 1935: bred vectors relative to the (a) first, (c) second and (e) third targeted observation. Panels (b), (d) and (f) show the same fields, after regionalization. In all fields the black dot marks the maximum of the structure, i.e. the target position for the observation. The field shown is the targeted interface elevation, that between layers 3 and 4 in the three cases.

Note here the theoretical importance of being able to predict structures that will be observed, and that the correlation between the "dynamical" analysis increment and the forecast error is also high in deeper layers, as shown in Fig. 7b. This means that the same tridimensional structure is present in the forecast error and in the forced bred vector. There is no way this could be obtained, for dynamic, evolving, nonlinear systems, with analysis schemes based on stationary estimates of error covariances.

Notice, again, the complex vertical structures of the signals present: the structure located at about $11 \mathrm{E}, 38 \mathrm{~N}$ appears rotated when the surface fields are compared with deeper 
(a)

day 1935 forecast error \& analysis increment L3/4 elevation (m)

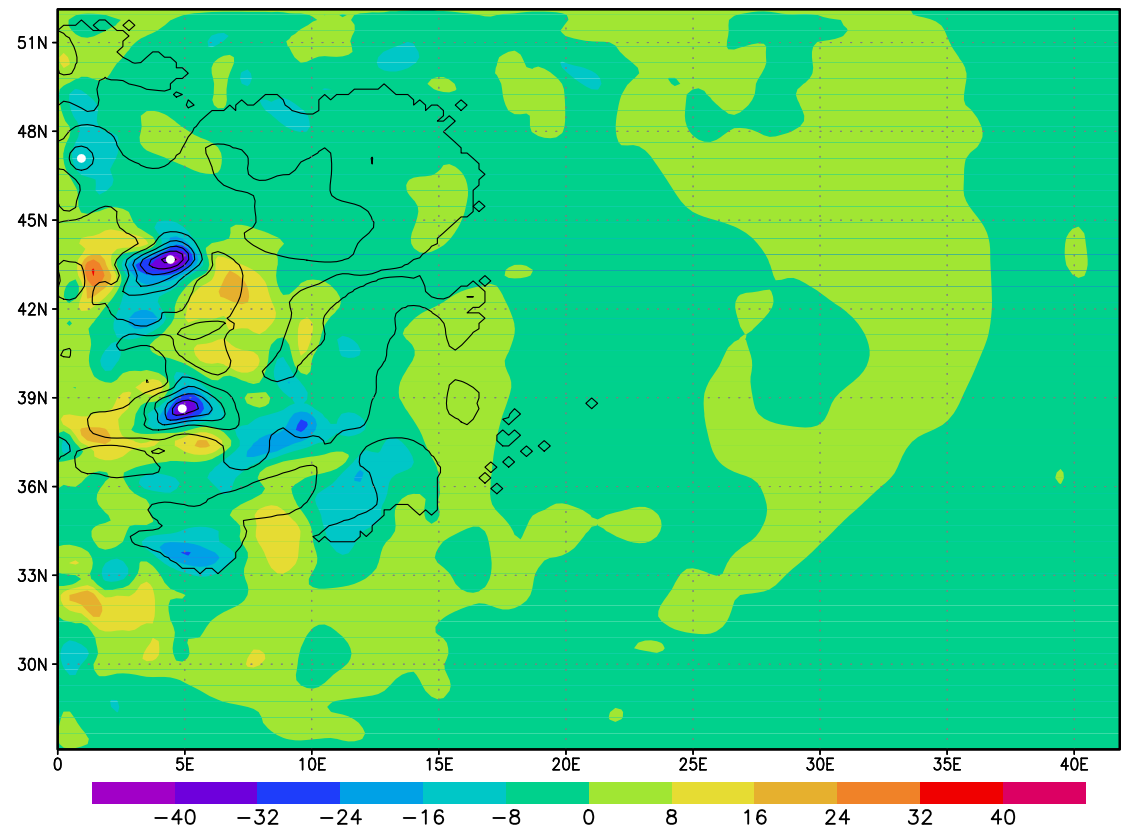

(b)
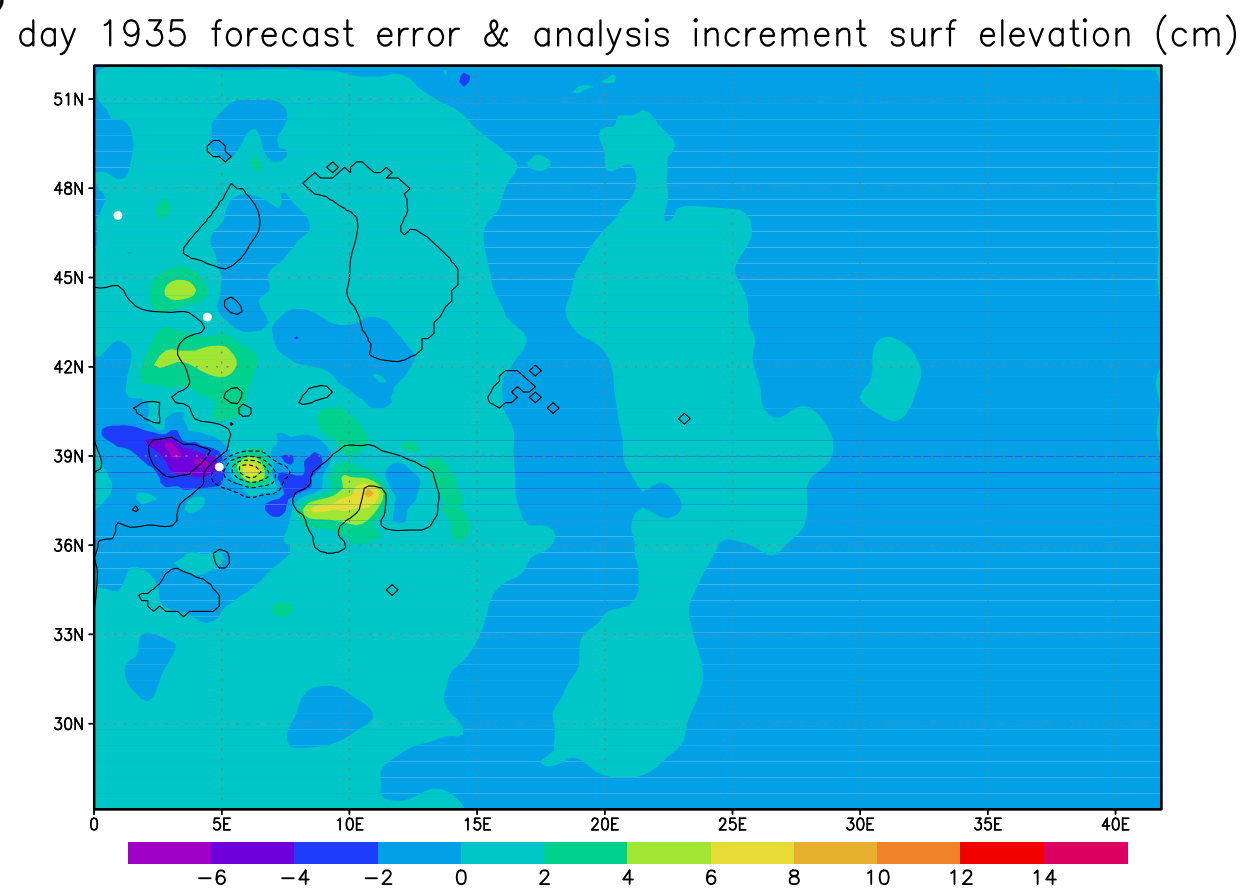

Fig. 6. Day 1935. Forecast error (colour shading) and analysis increment (contour lines): (a) elevation of the interface between layers 3 and 4; (b) sea surface elevation. The white dots mark the horizontal positions of the three adaptive observations, targeted at the interface between layers 3 and 4 .

fields; a dipole in the deeper field at about $2 \mathrm{E}, 43 \mathrm{~N}$ appears as an isolated maximum at the surface.

In correspondence with this last structure, the correlation is even better in the deeper fields than at the observed surface field. Since here the correlation between analysis increment and forecast error is high even far (vertically) from the obser- vations, the assimilation is successful. However, for AUS as well as for other ensemble-based assimilation schemes such as EnKF and SEEK, a dangerous situation may arise, especially when only a fixed observational network is used. If an unstable structure is only marginally detected by observations that are located far from its maximum (say in mass 
(a)

(a)

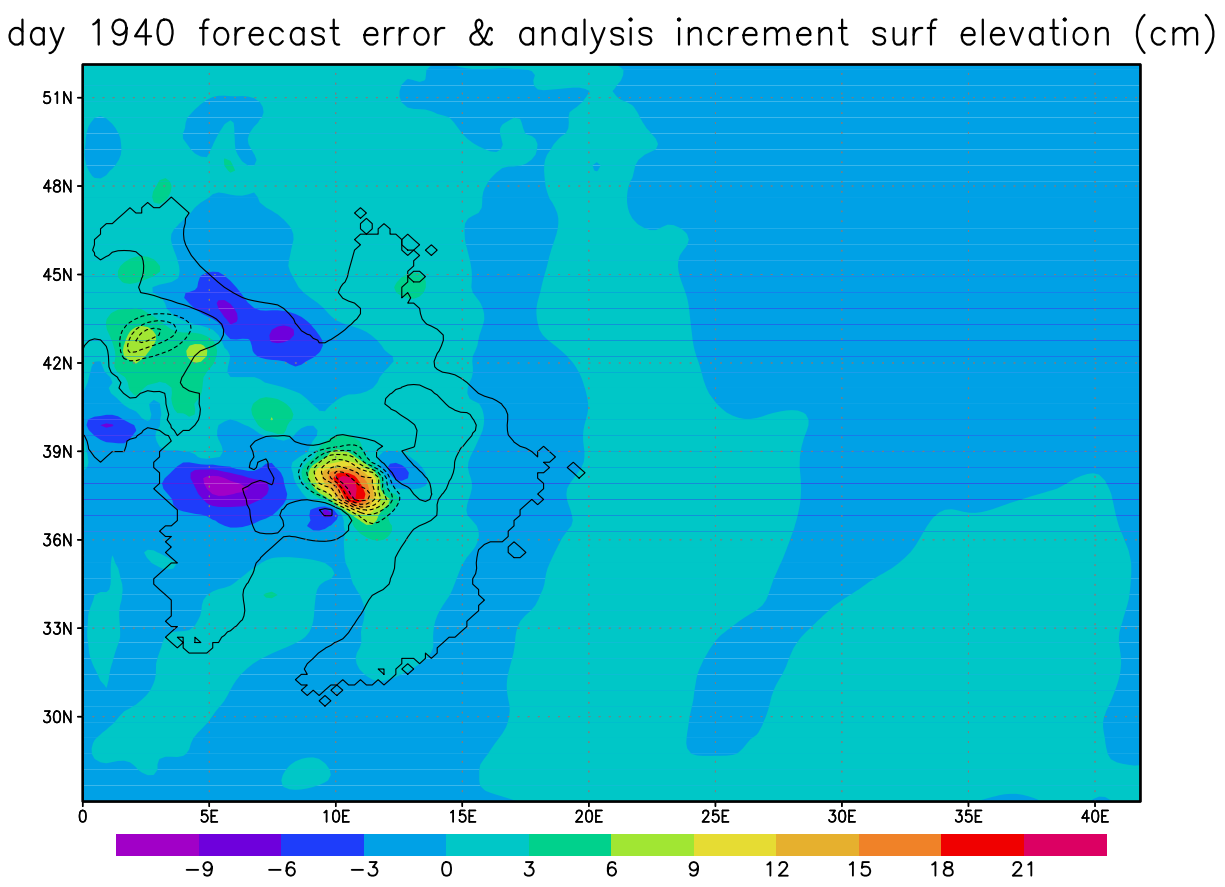

(b)

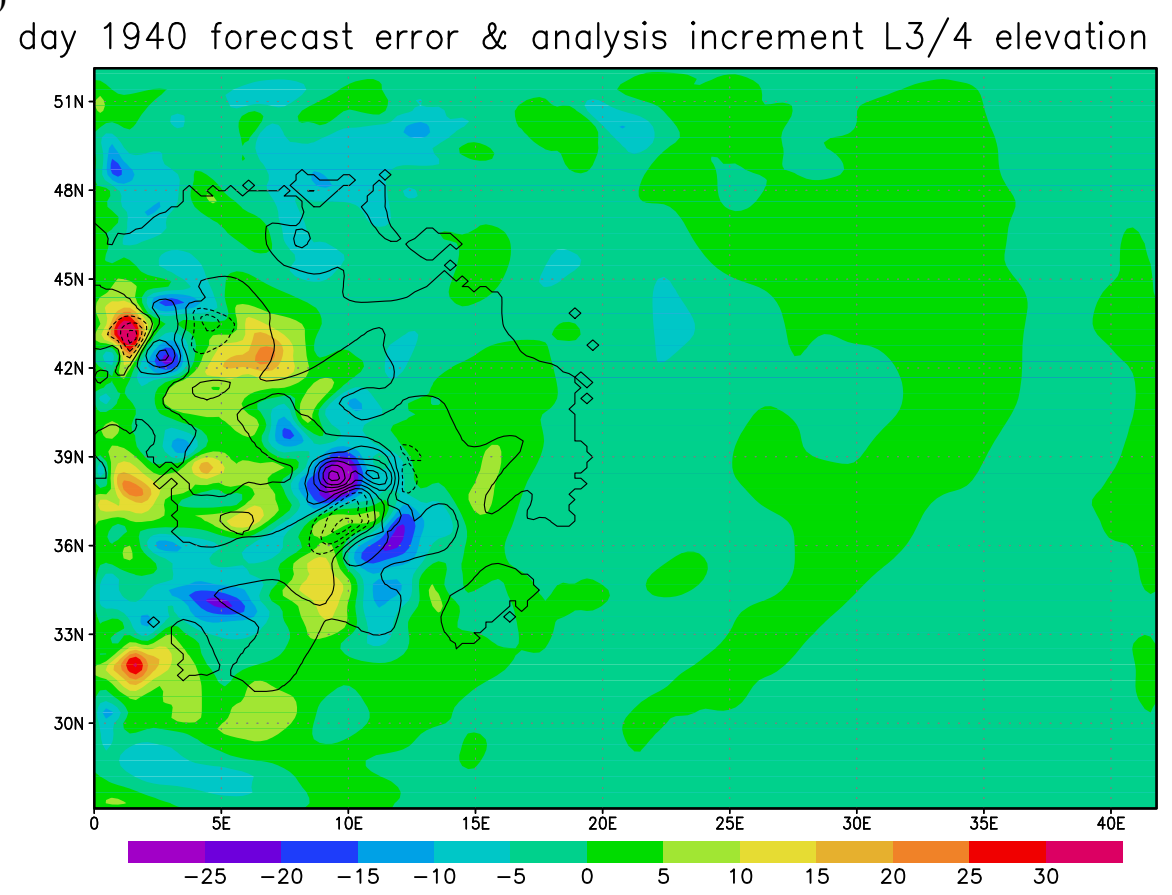

Fig. 7. Day 1940. Forecast error (colour shading) and AUS analysis increment (contour lines): (a) sea surface elevation. (b) elevation of the interface between layers 3 and 4 .

or energy), then the amplitude of the forecast error component on this mode may be inaccurately estimated, (for example if other, independent structures exist in the same region, or by effect of observational error) and the unstable component, instead of being reduced, may even be amplified by the assimilation. This clarifies the reasons why the "selection" procedure (besides "regionalization" that acts in the horizontal directions) discussed in Sect. 4 has been implemented for standard observations.

\section{Conclusions}

By means of BDAS it is possible to obtain an accurate estimate of the local structures present in the unstable vectors, and that characterize the growing components of errors in cyclic observation-analysis-forecast systems of the ocean and of the atmosphere. By means of AUS it is possible and it is worthwhile - to use observations to eliminate the unstable structures present in the forecast error at analysis 
time. In the present and in previous works (TU04, UTC05 and CTU06), BDAS and AUS have been successfully applied to atmospheric and oceanic systems that are different for complexity and degree of approximation, in presence of different standard observational networks and adaptive observations.

With particular regard to the present work, results have been obtained in a perfect model and perfect observations settings. Generally speaking, experiments done in perfect conditions can produce deceivingly encouraging results, so we caution the reader against too optimistic an interpretation of the presented results.

While a thorough investigation of the effects of model error is beyond the scope of the present work, it is clear that a large model error could degrade the estimation of the unstable directions. In the present work the perfect model choice has allowed to highlight the role of chaotic instability in the growth of errors and on how it can be neutralized. On the other hand, as the error growth is linked to chaotic instability even for noisy systems, the estimation of the unstable directions also appears promising for realistic, imperfect models, with real, noisy data. Results obtained with a QuasiGeostrophic atmospheric model in the presence of observational error (CTU06) are encouraging.

Using imperfect observations may lead to errors in the estimate of the amplitude of the corrections. This means that the unstable components cannot be eliminated, but only reduced, by the assimilation. This problem can be overcome by using more observations for each structure, thus obtaining some error cancellation. Alternatively, a reduction of error can be obtained by increasing the observational frequency.

All considerations on observational errors may be considered part of the more general problem concerning the ability of the observational network to detect the unstable structures. As predicted by the theory and confirmed by the experiments, it is important that the observational network, in its fixed and adaptive components, is able to detect the unstable structures that are present in the system, and that migrate, while growing, through the domain, depending on the characteristics of the flow.

The stabilization obtained by BDAS-AUS with adaptive observations results in a remarkable reduction of errors for all the variables and all layers, and in a reduction of their tendency to grow during the model integration from the analysis state to the next forecast state. Generally speaking, when an assimilation scheme, by employing dynamically evolving covariances, is able to stabilize the system and to control the error growth, this means that a suitable estimate of the unstable structures has been obtained and that the observational network has the ability of detecting them. On the other hand, one or both of these two key elements are most probably missing when errors show episodes of uncontrolled growth.

Finally, the study of the horizontal distribution of adaptive observations, in relation to the average error fields at the different levels, has led to the following conclusion. The use of adaptive observations has been crucial to detect unstable structures that grow in deep layers, particularly in western boundary current regions, before they emerge in layers closer to the surface and manifest themselves in instabilities associated to the eastward jet, its meanders and its eddies. The deep layers of the ocean may appear to be less active than those near the surface, but, due to fluctuations of the former, the dynamics of the latter can be destabilized.

Acknowledgements. We acknowledge the contribution of A. Carrassi to the elaboration of BDAS and AUS and warmly thank him for the discussions concerning this manuscript. We have greatly benefited of the enlightening comments by E. Kalnay. The MICOM version and configuration used in this work have been obtained from SHOM, Toulouse. The initial part of this work has been financially supported by the projects EPSHOM-UBO: 00.87.118.00.470.29.25 and CA2003/03/CMO.

Edited by: O. Talagrand

Reviewed by: E. Kalnay and another referee

\section{References}

Bennett, A. F.: Inverse methods in physical oceanography, Cambridge University Press, 1992.

Bennett, A. F.: Inverse Modeling of the Ocean and Atmosphere, Cambridge University Press, 2002.

Bergot, T., Hello, G., Joly, A., and Marlardel, S.: Adaptive observations: a feasibility study, Mon. Wea. Rev., 127, 743-765, 1999.

Berliner, L. M., Lu, Z.-Q., and Snyder, C.: Statistical design for adaptive weather observations, J. Atmos. Sci., 56, 2536-2552, 1999.

Bishop, C., Etherton, B. J., and Majumdar, S. J.: Adaptive sampling with the Ensemble Transform Kalman Filter. Part I: theoretical aspects, Mon. Wea. Rev., 129, 420-436, 2001.

Bleck, R.: Simulation of Coastal Upwelling Frontogenesis with an Isopycnic Coordinate Model, J. Geophys. Res., 83C, 6163-6172, 1978.

Brasseur, P., Ballabrera-Poy, J., and Verron, J.: Assimilation of altimetric data in the mid-latitude oceans using the Singular Evolutive Extended Kalman Filter with an eddy-resolving, primitive equation model, J. Mar. Syst., 22, 269-294, 1999.

Buehner, M. and Zadra, A.: Impact of flow-dependent analysiserror covariance norms on extratropical singular vectors, Quart. J. Roy. Meteorol. Soc., 132, in press, 2006.

Burgers, G., Van Leeuwen, P. J., and Evensen, G.: Analysis scheme in the ensemble Kalman filter, Mon. Wea. Rev., 126, 1719-1724, 1998.

Cooper, M. and Haines, K.: Altimetric Assimilation with Water Property Conservation, J. Geophys. Res., 101C, 1059-1077, 1996.

Corazza, M., Kalnay, E., Patil, D. J., Ott, E., Yorke, J. A., Hunt, B. R., Szunyogh, I., and Cai, M.: Use of the breeding technique in the estimation of the background covariance matrix for a quasi-geostrophic model, in AMS Symposium on Observations, Data Assimilation and Probabilistic Prediction, 13-17 January 2002, Orlando, Florida, 154-157, 2002.

Corazza, M., Kalnay, E., Patil, D. J., Yang, S.-C., Morss, R., Cai, M., Szunyogh, I., Hunt, B. R., and Yorke, J. A.: Use of the breeding technique to estimate the structure of the analysis "errors of the day", Nonlin. Processes Geophys., 10, 233-243, 2003,

SRef-ID: 1607-7946/npg/2003-10-233. 
De Mey, P. and Benkiran, M.: A multivariate reduced-order optimal interpolation method and its application to the mediterranean basin-scale circulation, in: Ocean forecasting, conceptual basis and applications, edited by: Pinardi, N. and Woods, J. D., Springer-Verlag, 472 p., 2002.

ECCO Consortium: The consortium for Estimating the Circulation and Climate of the Ocean (ECCO) - Science goals and task plan, Tech. rep., ECCO N. 1, Scripps Institution of Oceanography, http://www.ecco-group.org, 1999.

Ehrendorfer, M. and Tribbia, J. J.: Optimal Prediction of forecast error covariances through singular vectors, J. Atmos. Sci., 54, 286-313, 1997.

Evensen, G.: Sequential data assimilation with a nonlinear quasigeostrophic model using Monte-Carlo methods to forecast error statistics, J. Geophys. Res., 99, 10 143-10 162, 1994.

Evensen, G.: The Ensemble Kalman Filter: Theoretical Formulation and Practical Implementations, Ocean Dynamics, 53, 343367, 2003.

Evensen, G.: Sampling strategies and square root analysis schemes for EnKF, Ocean Dynamics, 54, 539-560, 2004.

Fourrié, N., Marchal, D., Rabier, F., Chapnik, B., and Desroziers, G.: Impact study of the 2003 North Atlantic THORPEX Regional Campaign, Quart. J. Roy. Meteorol. Soc., 132, in press, 2006.

Ghil, M.: Meteorological data assimilation for oceanographers. Part I: description and theoretical framework, Dynam. Atmos. Oceans, 13, 171-218, 1989.

Ghil, M.: Advances in sequential estimation for atmospheric and oceanic flows, J. Meteorol. Soc. Japan, 75, 289-304, 1997.

Kalnay, E.: Atmospheric Modeling, Data Assimilation and Predictability, Cambridge University Press, 2003.

Köhl, A. and Stammer, D.: Optimal Observations for Variational Data Assimilation, J. Phys. Oceanogr., 34, 529-542, 2004.

Lorenz, E. N. and Emanuel, K. A.: Optimal Sites for Supplementary Weather Observations: Simulation with a Small Model, J. Atmos. Sci., 55, 399-414, 1998.

Mourre, B.: Étude de configuration d'une constellation de satellites altimétriques pour l'observation de la dynamique océanique côtière, Ph.D. thesis, Université de Toulouse III, France, 2004.

Palmer, T. N., Gelaro, R., Barkmeijer, J., and Buizza, R.: Singular vectors, metrics, and adaptive observations, J. Atmos. Sci., 55, 633-653, 1998.
Pham, D. T.: Stochastic methods for sequential data assimilation in strongly nonlinear systems, Mon. Wea. Rev., 129, 1194-1207, 2001.

Rotunno, R. and Bao, J. W.: A Case Study of Cyclogenesis Using a Model Hyerarchy, Mon. Wea. Rev., 124, 1051-1066, 1996.

Szunyogh, I., Toth, Z., Morss, R. E., Majumdar, S. J., Etherton, B. J., and Bishop, C. H.: The effect of targeted dropsonde observations during the 1999 winter storm reconnaissance program, Mon. Wea. Rev., 128, 3520-3537, 2000.

Szunyogh, I., Toth, Z., Zimin, A., Majumdar, S. J., and Persson, A.: Propagation of the effect of targeted observations: the 2000 winter storm reconnaissance program, Mon. Wea. Rev., 130, 1144 $1165,2002$.

Talagrand, O. and Courtier, P.: Variational assimilation of meteorological observations with the adjoint vorticity equation. I: theory, Quart. J. Roy. Meteorol. Soc., 113, 1311-1328, 1987.

Toth, Z. and Kalnay, E.: Ensemble Forecasting at NCEP: the Breeding Method, Mon. Wea. Rev., 125, 3297-3318, 1997.

Trevisan, A. and Pancotti, F.: Periodic Orbits, Lyapunov Vectors, and Singular Vectors in the Lorenz System, J. Atmos. Sci., 55, 390-398, 1998.

Trevisan, A. and Uboldi, F.: Assimilation of Standard and Targeted Observations in the Unstable Subspace of the ObservationAnalysis-Forecast Cycle System, J. Atmos. Sci., 61, 103-113, 2004.

Uboldi, F. and Kamachi, M.: Time-space weak constraint data assimilation with nonlinear models, Tellus, 52A, 412-421, 2000.

Uboldi, F., Trevisan, A., and Carrassi, A.: Developing a Dynamically Based Assimilation Method for Targeted and Standard Observations, Nonlin. Processes Geophys., 12, 149-156, 2005,

SRef-ID: 1607-7946/npg/2005-12-149.

Whitaker, J. S. and Hamill, T. M.: Ensemble Data Assimilation without Perturbed Observations, Mon. Wea. Rev., 130, 19131924, 2002.

Wunsch, C.: The Ocean Circulation Inverse Problem, Cambridge University Press, 1996.

Zang, X. and Malanotte-Rizzoli, P.: A comparison of Assimilation Results from the Ensemble Kalman Filter and a Reduced-Rank Extended Kalman Filter, Nonlin. Processes Geophys., 10, 477491, 2003,

SRef-ID: 1607-7946/npg/2003-10-477. 